\title{
IMPROVED SEMI-PARAMETRIC MEAN TRAJECTORY MODEL USING DISCRIMINATIVELY TRAINED CENTROIDS
}

\author{
Ran Xu, Jielin Pan, Yonghong Yan \\ ThinkIT Speech Laboratory \\ Institute of Acoustics, Chinese Academy of Sciences \\ No. 21, North 4th Ring Middle Road, Beijing
}

\begin{abstract}
In order to alleviate the limitation of "state output probability conditional independence" assumption held by Hidden Markov models (HMMs) in speech recognition, a discriminative semi-parametric trajectory model was proposed in recent years, in which both means and variances in the acoustic models are modeled as time-varying variables. The timevarying information is modeled as a weighted contribution from all the "centroids", which can be viewed as the representation of the acoustic space. In previous literatures, such centroids are often obtained by clustering the Gaussians in the baseline acoustic models to some reasonable number or by training a baseline model with fewer Gaussian components. The centroids obtained in this way are maximum likelihood estimation of the acoustic space, which are relatively weak in discriminability compared to the discriminatively trained acoustic models. In this paper, we proposed an improved semi-parametric mean trajectory model training framework, in which the centroids are first discriminatively trained by minimum phone error criterion to provide a more discriminative representation of the acoustic space. This method was evaluated on the Mandarin digit string recognition task. The experimental result shows that our proposed method improves the recognition performance by a relative string error rate reduction of $7.5 \%$ compared to the traditional discriminative semi-parametric trajectory model, and it outperforms the baseline acoustic model trained with maximum likelihood criterion by a relative string error rate reduction of $28.6 \%$.
\end{abstract}

Index Terms - Speech recognition, trajectory model, discriminative training, minimum phone error

\section{INTRODUCTION}

Hidden Markov models (HMMs) [1] are the most commonly used acoustic models for the state-of-the-art speech recognition systems. However, the "state output probability conditional independence" assumption underlying HMM fails to model the correlations among speech frames. In order to alleviate this limitation, a number of modeling approaches are proposed by modeling the HMM parameters as time-dependent variables. They are all known as trajectory models $[2,3,4,5]$. Although they are well-defined in theory, training these models with maximum likelihood criterion yields little success in Large Vocabulary Continuous Speech Recognition (LVCSR) [10].

The success of discriminative training such as Maximum Mutual Information (MMI) [7] and Minimum Phone Error (MPE) [8] in LVCSR systems makes it possible to train trajectory models with discriminative criteria. The feature space MPE (fMPE) training [11] proposed in 2005 was originally presented as training a feature transformation matrix to add time-varying information to the original features. The latter literature presented a semi-parametric trajectory model framework [10], in which fMPE is interpreted as a kind of semi-parametric trajectory model, in which only mean parameters are modeled as time-varying variables. This is denoted as "semi-parametric mean trajectory model" in this paper.

In the semi-parametric mean trajectory model, the mean vectors are modeled as a time-varying function of the location of the current observation in the acoustic space. In order to do this, a representation of the acoustic space need to be defined. In the previous literatures, it employs a series of centroids, which are obtained by clustering the Gaussians in the baseline acoustic models to a reasonable number or by directly training a baseline model with fewer Gaussian components $[10,11,12]$. The centroids can be considered as a vector-quantization (VQ) codebook representing the acoustic space, which leads to the interpretation of such trajectory model as a semi-parametric model [10].

The centroids play an important role in modeling the time variation for mean vectors. Since they are appropriate representation of the real acoustic space, a more discriminative and accurate series of centroids may help to model a more accurate mean trajectory in the real acoustic space. Based on such idea, we proposed an improved semi-parametric mean trajectory model by using discriminatively trained centroids, in which the centroids are first trained with minimum phone error criterion. The method is evaluated and compared to the traditional semi-parametric mean trajectory model on the Mandarin digit string recognition task. The evaluation result 
shows that our proposed method can further improve the performance of semi-parametric mean trajectory model with a significant error rate reduction.

The rest of this paper is organized as follows. Section 2 reviews the theoretical basis of the semi-parametric mean trajectory model. Section 3 presents our improved training framework using discriminatively trained centroids. In section 4 the setup for experiments and experimental results are discussed. Conclusions are given in section 5.

\section{SEMI-PARAMETRIC MEAN TRAJECTORY MODEL}

According to [10], the time-varying mean vector of Gaussian component $m$ in state $s$ at time $t$ can be formulated as follows:

$$
\mu_{s m t}=A_{t} \mu_{s m}+b_{t}
$$

where $A_{t}$ is a time-dependent linear transformation and $b_{t}$ denotes a time-dependent bias vector to the mean. To simplify the question, $A_{t}$ is usually taken as an identity matrix, while $b_{t}$ is modeled as a weighted contribution from all the centroids in the acoustic space, which is defined as:

$$
b_{t}=\sum_{i=1}^{n} h_{i}(t) b^{(i)}
$$

where $h_{i}(t)$ denotes the contribution weight from the $i$ th centroid at time $t$ and $n$ is the total number of the centroids. $b^{(i)}$ is the $i$ th column of the linear transformation matrix $B$. It is a $d \times n$ matrix where $d$ denotes the dimension of the features. To be more precise, the time-varying mean vector in the $j$ th dimension can be written as:

$$
\mu_{s m t j}=\mu_{s m j}+b_{t j}
$$

Let $C_{i}$ represent the $i$ th centroid modeled as a Gaussian distribution, the weight $h_{i}(t)$ is calculated as the posterior probability of the $i$ th centroid given the observation feature vector $o_{t}$ :

$$
h_{i}(t)=P\left(C_{i} \mid o_{t}\right)=\frac{P\left(o_{t} \mid C_{i}\right) P\left(C_{i}\right)}{\sum_{j=1}^{n} P\left(o_{t} \mid C_{j}\right) P\left(C_{j}\right)}
$$

where $P\left(C_{i}\right)$, the prior probability of the $i$ th centroid, is assumed to be equal [10]. $P\left(o_{t} \mid C_{i}\right)$ is the likelihood of $C_{i}$ given the observation $o_{t}$ :

$P\left(o_{t} \mid C_{i}\right)=\frac{1}{\sqrt{(2 \pi)^{d}\left|\Sigma_{i}\right|}} \exp \left\{-\frac{1}{2}\left(o_{t}-\mu_{i}\right)^{T} \Sigma_{i}^{-1}\left(o_{t}-\mu_{i}\right)\right\}$

According to the above formulation, the mean vector is modeled as a time-varying parameter changing with the position of the current observation in the acoustic space, which tracks the observation as a smooth trajectory. The transformation matrix $B$ is discriminatively estimated with the MPE objective function. The detailed optimization procedures are well discussed in $[10,11]$.

\section{IMPROVED TRAINING FRAMEWORK USING DISCRIMINATIVELY TRAINED CENTROIDS}

$h_{i}(t)$ calculated by equation (4) is an important variable used throughout all the differential calculations in the MPE objective function optimization procedure. The centroids play as the reference acoustic space to provide posterior probability of the current observation. In the previous literatures, it is often obtained from the Gaussians in the baseline MLE models without their weight information, which forms an MLE model representation of the real acoustic space. Since MPE model achieves much lower error rate on various speech recognition tasks, we believe MPE model will be a more accurate representation of the real acoustic space and will help to better model the mean trajectory in the semi-parametric trajectory models. Thus, in our improved training framework, centroids are first discriminatively trained with MPE criterion to provide a more accurate and discriminative representation of the acoustic space.

Let $\mu_{c, j}$ and $\sigma_{c, j}^{2}$ denote the mean and variance in the $j$ th dimension of centroid $c$, under MPE criterion , they are updated by [8]:

$$
\begin{aligned}
\hat{\mu}_{c, j} & =\frac{\left\{\theta_{c, j}^{\text {num }}(O)-\theta_{c, j}^{\text {den }}(O)\right\}+D \mu_{c, j}}{\left\{\gamma_{c, j}^{\text {num }}-\gamma_{c, j}^{\text {den }}\right\}+D}, \\
\hat{\sigma}_{c, j}^{2} & =\frac{\left\{\theta_{c, j}^{\text {num }}\left(O^{2}\right)-\theta_{c, j}^{\text {den }}\left(O^{2}\right)\right\}+D\left(\sigma_{c, j}^{2}+\mu_{c, j}^{2}\right)}{\left\{\gamma_{c, j}^{\text {num }}-\gamma_{c, j}^{\text {den }}\right\}+D}-\hat{\mu}_{c, j}^{2}
\end{aligned}
$$

where $D$ is a constant controlling the convergence speed. Small $D$ results in a larger step size and a faster convergence speed. However, using small values of $D$ typically results in oscillatory behavior, which may cause unstable training. In practice a lower bound of $D$ is the value which ensures all variances remaining positive [7]. Details of other parameters in equation (6) and how to calculate them are discussed in [9].

Fig.1 illustrates the training framework presented in our system. The parameter estimation follows the same routine described in $[10,11]$ except that the $h_{i}(t)$ is calculated on the discriminatively trained centroids. The parameter estimation involves calculating those differentials associated with the transformation matrix (dynamic parameters and direct differentials) and those related with static means and variances (static parameters and indirect differentials) [10]. In implementation a training iteration for the semi-parametric mean trajectory model involves 3 training passes. The first-pass of MPE accumulates the overall MPE statistics for calculating the indirect differentials. The second-pass of MPE calculates the direct and indirect statistics for updating the linear transformation matrix $B$. After the matrix has been updated, origi- 


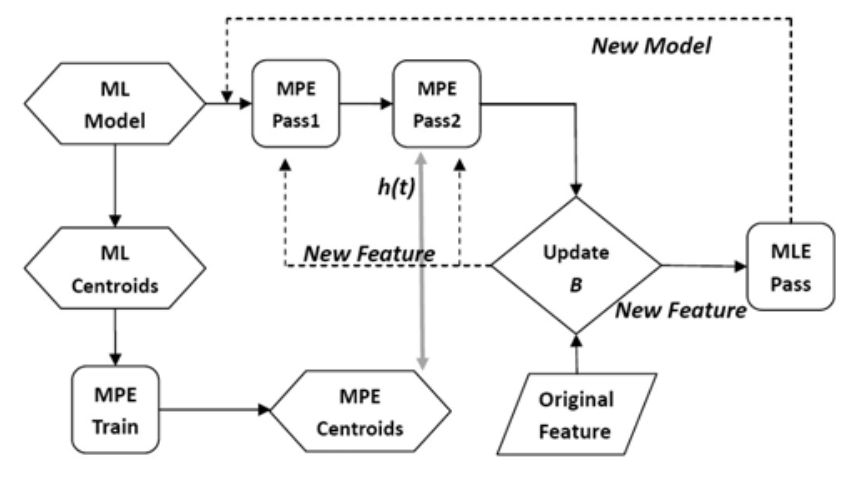

Fig. 1. Illustration of the training procedures for semiparametric mean trajectory model

nal feature set is converted to new feature set with mean offset reflected by the trajectory model. A final MLE pass updates the model static parameters with the new feature. Then the new feature and the new model start the next iteration from MPE Pass1. Typically three or four passes of such iterations can produce the best result.

\section{EXPERIMENTAL RESULTS}

The experimental results presented in this section are based on a Mandarin digit string recognition task. Digit string recognition is a pure acoustic modeling problem without the interference of language models, which can explicitly reflect the accuracy of acoustic modeling.

\subsection{Experimental setup}

The feature used for the acoustic modeling consists of 12 perceptual linear prediction (PLP) coefficients with the C0 energy term and their first and second derivatives, which forms a feature vector of 39 dimensions. Heteroscedastic linear discriminant analysis (HLDA) [6] is then applied to project the feature down to 24 dimensions.

The baseline acoustic models are gender-independent cross-word triphones with 3-state left-to-right topology. A robust state clustering with two-level phonetic decision trees are used. 611 shared states are empirically determined with 8 Gaussians per state. The models are trained using 62 hours of digit string utterances.

For the semi-parametric mean trajectory models, the training lattices for MPE are generated by recognizing the whole training set with N-best options [7, 8]. 4888 Gaussians in the baseline MLE models are directly taken out as the centroids for the baseline trajectory models (System BMT in Table 1 in section 4.2). While in our proposed training framework, the baseline MLE models are further trained with MPE criterion before they are used as centroids (System DCMT in Table 1 in section 4.2).

All experiments are performed on a Mandarin digit string test set consisting of 1,000 utterances. The shortest string contains 8 digits and the longest string contains 11 digits. The evaluation criterion for the test is String Error Rate (SER), under which an utterance is taken as correct recognition result only when all the digits in the string are correctly recognized.

\subsection{Evaluation results}

There are four systems evaluated as shown in Table 1. The MLE system is the baseline models trained with the maximum likelihood estimation criterion. Other systems are trained starting from this model. The BMT system is the baseline mean trajectory models trained according to the framework proposed in $[10,11]$. The DCMT system is our proposed mean trajectory models trained with discriminatively trained centroids. The MPE system is the MLE models trained with MPE criterion, from which the discriminatively trained centroids are obtained. All discriminative models (BMT, DCMT and MPE) are trained through 4 iterations. Fig. 2 gives the SER curve of each system to provide better visualized comparison.

Table 1. SER (\%) comparisons of different systems

\begin{tabular}{|c||c|c|c|c|c|}
\hline Iteration & Base & 1 & 2 & 3 & 4 \\
\hline \hline MLE & 22.4 & - & - & - & - \\
BMT & 22.4 & 19.7 & 18.7 & 17.8 & 17.3 \\
DCMT & 22.4 & 18.4 & 16.9 & 16.4 & 16.0 \\
MPE & 22.4 & 20.4 & 19.5 & 18.5 & 18.5 \\
\hline
\end{tabular}

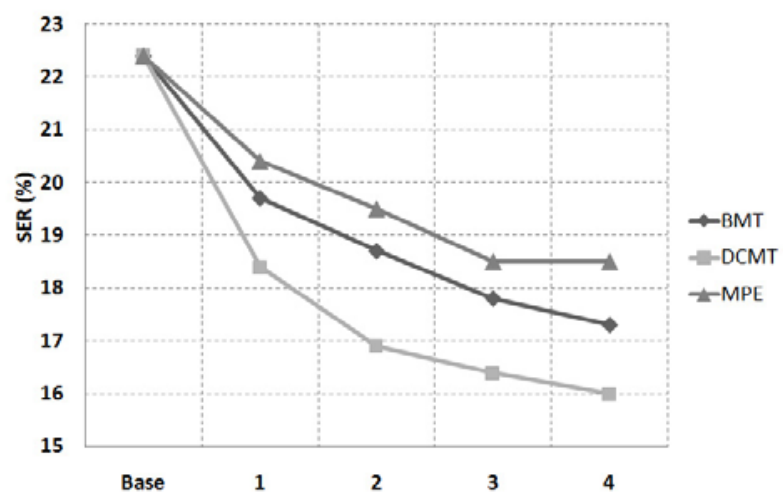

Fig. 2. Changes in SER with increasing training iterations

Table 1 and Fig. 2 show that our proposed semi-parametric mean trajectory model training framework using discriminatively trained centroids achieves the best performance on the 


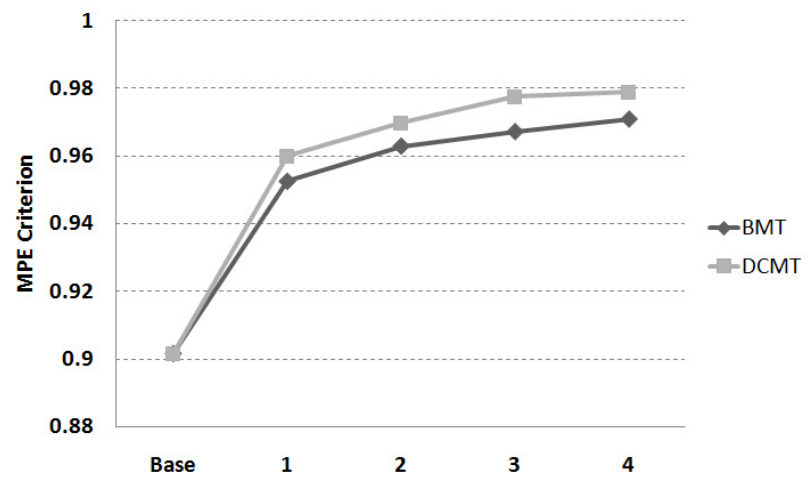

Fig. 3. Change in MPE criterion with increasing training iterations

test set with the lowest SER of $16.0 \%$. It outperforms the baseline MLE model by $28.6 \%$ relative SER reduction and outperforms the traditional semi-parametric mean trajectory model (BMT) by $7.5 \%$ relative. The improvement is given by employing a series of more discriminative centroids to represent the real acoustic space. It can be seen that the MPEtrained centroids yield a much lower SER compared to the baseline MLE model (17.4\% relative SER reduction). The MPE criterion increasing curve shown in Fig. 3 indicates that our proposed method can effectively increase the MPE criterion on the training set. Since the semi-parametric mean trajectory model is trained with MPE criterion, the increase of objective function value reflects the discriminative efficiency of the training models on the training set. Under the same condition, a higher MPE criterion may result in better performance on the test set.

\section{CONCLUSIONS}

This paper proposed an improved training framework using discriminatively trained centroids instead of using those derived from the baseline MLE models for semi-parametric mean trajectory model training. In this model, the state output probability density function is represented by a Gaussianmixture model (GMM) where the Gaussian mean vector varies with time. The time dependency is modeled as a smoothed function of tracking the observation position in the acoustic space, which is represented by a series of centroids. Discriminatively trained centroids provide a much more discriminative representation of the acoustic space and result in a better trajectory model. Our evaluation result shows that the proposed method can further improve the performance of traditional mean trajectory models by $7.5 \%$ relative SER reduction on the long Mandarin digit string recognition task.

\section{REFERENCES}

[1] L.A. Rabiner, "A tutorial on hidden Markov models and selective applications in speech recognition," Proc. IEEE, vol. 77, pp. 257-286, 1989.

[2] A-V.I. Rosti and M.J.F. Gales, "Switching linear dynamical systems for speech recognition," Technical Report, CUED/F-INFENG/TR461, University of Cambridge, 2003.

[3] M. Ostendorf, V. Digalakis and O. Kimball, "From HMM's to segment models: a unified view of stochastic modeling for speech recognition," IEEE Transactions on Speech and Audio Proceeding 4 (5), pp. 360-378, 1996.

[4] J.A. Bilmes, "Buried Markov models for speech recognition," Proc ICASSP, pp. 713-716, 1999.

[5] K. Tokuda, H. Zen and T. Kitamura, "Trajectory modeling based on HMMs with the explicit relationship between static and dynamic features," Proc. European Conference on Speech and Communication Technology, pp. 865-868, 2003.

[6] N. Kumar, Investigation of Silicon Auditory Models and Generalization of Linear Discriminant Analysis for Improved Speech Recognition, $\mathrm{PhD}$ thesis, Johns Hopkins University, 1997.

[7] V. Valtchev, J.J. Odell and P.C. Woodland, "MMIE training of large vocabulary recognition systems," Speech Communication , vol. 22, pp. 303-314, 1997.

[8] Daniel Povey and P.C. Woodland, "Minimum phone error and I-smoothing for improved discriminative training," Proc. ICASSP, 2002.

[9] Daniel Povey, Discriminative Training for Large Vocabulary Speech Recognition, $\mathrm{PhD}$ thesis, University of Cambridge, 2004.

[10] K.C. Sim and M.J.F. Gales, "Discriminative semiparametric trajectory model for speech recognition," Computer speech and language, vol. 21, pp. 669-687, 2007.

[11] Daniel Povey, Brian Kingsbury, Lidia Mangu, George Saon, Hagen Soltau and Geoffrey Zweig, "fMPE: Discriminatively trained features for speech recognition," Proc. ICASSP, 2005.

[12] Daniel Povery, "Improvements to fMPE for discriminative training of features," Interspeech 2005, 2005. 